Pacific Journal of Mathematics

HYPERSPACES OF GRAPHS ARE HILBERT CUB

Rd Miles SChorl AND JaMes Edward West 


\title{
HYPERSPACES OF GRAPHS ARE HILBERT CUBES
}

\author{
R. M. Schori AND J. E. West
}

\begin{abstract}
The authors prove that $2^{\Gamma}$ is a Hilbert cube where $\Gamma$ is any nondegenerate, finite, connected graph and $2^{\Gamma}$ is the space of nonvoid closed subsets of $\Gamma$ metrized with the Hausdorff metric. This extends their result that $2^{I}$ is a Hilbert cube. They also prove corresponding theorems for local dendrons $D$ as well as for the space of subcontinua $C(D)$ of $D$.
\end{abstract}

1. Introduction. In [9] the authors outlined their proof that $2^{I}$, the space of nonvoid, closed subsets of $I=[0,1]$ metrized with the Hausdorff metric, is a Hilbert cube $Q$ and announced the main results concerning graphs in this paper. Here we give the complete proof, assuming that $2^{I}$ is a Hilbert cube, that $2^{\Gamma}$ is a Hilbert cube for any finite, connected graph $\Gamma$. We also prove that if $D$ is any local dendron, then $2^{D}$ is a Hilbert cube and prove some results about the space of subcontinua $C(D)$ of a local dendron $D$ that extend the results of [13].

In [10] the authors give a complete proof that $2^{I}$ is a Hilbert cube. This settled a conjecture raised by Wojdyslawski [16] in 1938 where he also asked if $2^{X}$ is a Hilbert cube for any nondegenerate Peano space $X$. The first author and D. W. Curtis have announced the proof of this latter conjecture in [5] as well as the theorem that says that $C(X)$ is always a $Q$-factor for any Peano space $X$, and $C(X)$ is a Hilbert cube iff $X$ is a nondegenerate Peano space that contains no free arcs. These results are strongly dependent upon the results of this paper. The complete proofs of the $2^{x}$ and $C(X)$ results appear in [6].

This paper assumes the $2^{I}$ result and not the techniques of the proof. The proofs given here use some of the fundamental results of infinite-dimensional topology, but if the reader takes these results, listed in $\S 2$, as axioms, then no previous knowledge of infinite-dimensional topology is necessary for understanding this paper.

The authors thank D. W. Curtis for some useful suggestions concerning this paper.

2. Definitions and infinite-dimensional topology background. If $X$ is a compact metric space, then the Hausdorff metric $D$ on $2^{X}$ can be defined by

$$
D(A, B)=\inf \{\varepsilon>0: A \subset U(B, \varepsilon) \text { and } B \subset U(A, \varepsilon)\}
$$

where $U(C, \varepsilon)$ is the open $\varepsilon$-neighborhood of $C \subset X$. If $V$ is a subset 
of $X$, then $2_{V}^{X}$ is the subspace of $2^{X}$ consisting of all members of $2^{X}$ that contain $V$, and likewise for $C_{V}(X)$.

Let $Q$ denote the countable infinite product of $I$ with itself and define a Hilbert cube as any space homeomorphic $(\approx)$ to $Q$. A space $X$ is a $Q$-factor if $X \times Q \approx Q$. A $Q$-manifold is a separable metric space such that each point has an open neighborhood homeomorphic to an open subset of $Q$.

A map is a continuous function. If $X$ and $Y$ are homeomorphic compact metric spaces, then a map $f: X \rightarrow Y$ is a near-homeomorphism if for each $\varepsilon>0$ there exists a homeomorphism $h: X \rightarrow Y$ such that $d(f, h)<\varepsilon$. We say that $f: X \rightarrow Y$ stabilizes to a near-homeomorphism if $f \times i d: X \times Q \rightarrow Y \times Q$ is a near-homeomorphism. By a graph we will mean a 1-dimensional polyhedron with a specific triangulation.

R. D. Anderson's notion of $Z$-set [1] is extensively used in this paper and is one of the fundamental concepts in infinite-dimensional topology. There have been various definitions of $Z$-sets in the literature [1], [2], [4], and [7]. The following is the most convenient formulation for this paper.

Definition 2.1. A closed subset $A$ of a $Q$-factor $X$ is a $Z$-set in $X$ if for each $\varepsilon>0$ there exists a map $f: X \rightarrow X \backslash A$ such that $d(f, i d)<\varepsilon$.

We list below two well-known properties of $Z$-sets, the proofs of which are very easy. All spaces below are $Q$-factors.

2.2. $Z$-set Properties.

(a) If $A$ is a $Z$-set in $X$, then $A \times Y$ is a Z-set in $X \times Y$.

(b) Any finite union of $Z$-sets is a $Z$-set.

One of the important theorems in infinite-dimensional topology is the following theorem of Anderson. See [11] and [14] for generalizations.

2.3. First Sum Theorem [1]. If $A, B$, and $A \cap B$ are Hilbert cubes (Q-factors) and $A \cap B$ is a $Z$-set in $A$ ard in $B$, then $A \cup B$ is a Hilbert cube (Q-factor).

If $X$ and $Y$ are disjoint spaces, $A$ a closed subset of $X$, and $f: A \rightarrow Y$ a map, then the adjunction space of $f$, denoted $X \cup_{f} Y$, is $(X \cup Y) / R$, where $R$ is the equivalence relation on $X \cup Y$ generated by $a R f(a)$ for each $a \in A$. We say $X$ is attached to $Y$ by $f$. If $g: X \rightarrow Y$ is a map, then the mapping cylinder of $g$, denoted $M_{g}$, is the adjunction space $(X \times I) \bigcup_{g^{\prime}}, Y$ where $g^{\prime}: X \times\{0\} \rightarrow Y$ is defined 
by $g^{\prime}(x, 0)=g(x)$. The following is one of the basic theorems in the theory of $Q$-factors.

2.4. Mapping Cylinder Theorem [11] and [14]. Let $X$ and $Y$ be $Q$-factors and let $g: X \rightarrow Y$ be a map of $X$ into $Y$, then the mapping cylinder of $g, M_{g}$, is also a Q-factor. Furthermore, if $c: M_{g} \rightarrow$ $Y$ is the map defined by $c([x, t])=g(x)$, then $c$ stabilizes to a nearhomeomorphism.

An important corollary of this is the following.

2.5. The Attaching Theorem [10]. Let $X$ and $Y$ be Q-factors and let $A$ be a closed subset of $X$ that is a Z-set in $X$. If $f: A \rightarrow$ $Y$ is any map, then the adjunction space $X \cup_{f} Y$ is also a Q-factor.

A relative homeomorphism $f:(X, A) \rightarrow(Y, B)$ is a map of the pairs where $f \mid X \backslash A: X \backslash A \rightarrow Y \backslash B$ is a homeomorphism. The next remark is just a convenient alternative way of viewing adjunction spaces and will not be proved. Let all spaces below be compact metric.

REMARK 2.6. If $f:(X, A) \rightarrow(Y, B)$ is a relative homeomorphism, then $Y$ is homeomorphic to the adjunction space $X \cup_{g} B$ where $g=$ $f \mid A$.

The main tool of this paper is the following theorem.

2.7. Compactification Theorem [13]. Let $A$ be a closed subset of the space $X$ where

(1) $X$ is a $Q$-factor,

(2) $A$ is a Q-factor,

(3) $A$ is a $Z$-set in $X$, and

(4) $X \backslash A$ is a Q-manifold.

Then $X$ is a Hilbert cube.

The above theorem gives us conditions as to when the $Q$-manifold $X \backslash A$ can be compactified to be a Hilbert cube. We list the parts of the hypothesis because in practice the verification of each part will often be a separate result. To prove that $2^{\Gamma}$ is a Hilbert cube we will use the Compactification Theorem where $X=2^{\Gamma}$ and $A=C_{w}(\Gamma)$ for some vertex $w \in \Gamma$. In $\S 3$ we will prove that $2^{\Gamma}$ is a $Q$-factor and in $\S 4$ we will prove that $2^{r}$ and $C_{w}(\Gamma)$ satisfy the other three conditions. 
3. $2^{T}$ is a $Q$-factor. All of our results will be for the more general case $2_{V}^{r}$ where $V$ is any set of vertices (possibly empty) of a finite, connected graph. Note that if $V$ is empty, then $2_{V}^{\Gamma}=2^{\Gamma}$. We first prove two lemmas.

Let $\Gamma$ be a finite, connected, acyclic graph and let $V$ be any subset (possibly empty) of the vertices of $\Gamma$. Let $w$ be a vertex of $\Gamma$ which separates it and let $\Gamma_{1}, \cdots, \Gamma_{n}$ be the closure of the components of $\Gamma \backslash\{w\}$, denoting by $V_{i}$ the set $V \cap \Gamma_{i}, i=1, \cdots, n$. Suppose that $w \notin V$ and let $W=V \cup\{w\}$ and for each $i$, let $W_{i}=$ $V_{i} \cup\{w\}$. Let $X_{i}=\bigcup_{j=1}^{i}\left(2_{w_{j}}^{\Gamma_{j}} \times \prod_{k \neq j, k=1}^{i} 2_{V_{k}}^{\Gamma_{k}}\right)$.

LEMMA 3.1. $X_{n}$ is a Q-factor if the $2_{V_{j}^{j}}^{\Gamma_{j}}$ and $2_{W_{j}}^{\Gamma_{j}}$ are Q-factors.

Proof. For $i<n, X_{i+1}=2_{V_{i+1}}^{\Gamma_{i+1}} \times X_{i} \cup 2_{W_{i+1}}^{\Gamma_{i+1}} \times \Pi_{j=1}^{i} 2_{V_{j}^{j}}^{\Gamma}$, and $2_{V_{i+1}^{i+1}}^{\Gamma_{i+1}} \times$ $X_{i} \cap 2_{W_{i+1}}^{\Gamma_{i+1}} \times \Pi_{j=1}^{i} 2_{V_{j}^{j}}^{\Gamma_{j}}=2_{W_{i+1}}^{\Gamma_{i+1}} \times X_{i}$. Since $\Gamma$ is acyclic, $w$ is a free vertex of each $\Gamma_{i}$ and thus by a direct verification of the definition of a $Z$-set, each $2_{W_{i}}^{\Gamma_{i}}$ is a $Z$-set in $2_{V_{i}}^{\Gamma_{i}}$ and by $2.2(\mathrm{~b}), X_{i}$ is a $Z$-set in $\Pi_{j=1}^{i} 2_{V_{j}}^{\Gamma_{j}}$. Thus, by $2.2(\mathrm{a}), 2_{W_{i+1}}^{\Gamma_{i+1}} \times X_{i}$ is a $Z$-set in $2_{V_{i+1}^{i+1}}^{\Gamma_{i+1}} \times X_{i}$ and in $2_{W_{i+1}}^{\Gamma_{i+1}} \times \prod_{j=1}^{i} 2_{V}^{\Gamma_{j}^{j}}$. Note that a finite product of $Q$-factors is a $Q$-factor. Hence, by the First Sum Theorem, $X_{i+1}$ is a $Q$-factor if $X_{i}$ is one and since $X_{1}=2_{W_{1}}^{\Gamma_{1}}$ is a $Q$-factor by hypothesis, then $X_{n}$ is a $Q$-factor by induction and the proof is complete.

Let $Y_{n}$ be the set of all members of $2_{V}^{\Gamma}$ which meet each $\Gamma_{i}$.

LeMmA 3.2. $Y_{n}$ is a Q-factor if $2_{W}^{\Gamma}$ and the $2_{V_{j}^{j}}^{\Gamma_{j}}$ and $2_{W_{j}}^{\Gamma_{j}}$ are Qfactors.

Proof. If $F: \prod_{i=1}^{n} 2_{V_{i}}^{\Gamma_{i}} \rightarrow 2_{V}^{\Gamma}$ is defined by $F\left(A_{1}, \cdots, A_{n}\right)=A_{1} \cup \cdots$ $\cup A_{n}$, then $F:\left(\prod_{i=1}^{n} 2_{V_{\imath}}^{\Gamma_{i}}, X_{n}\right) \rightarrow\left(Y_{n}, 2_{W}^{\Gamma}\right)$ is a relative homeomorphism and hence $Y_{n}$ is homeomorphic to the adjunction space $\prod_{i=1}^{n} 2_{V_{i}}^{\Gamma_{i}} \mathbf{U}_{f} 2_{W}^{\Gamma}$ where $f=F \mid X_{n}$. Since each of $\prod_{i=1}^{n} 2_{V_{i}^{i}}^{r}, X_{n}$, and $2_{W}^{r}$ is a $Q$-factor and since $X_{n}$ is a $Z$-set in $\prod_{i=1}^{n} 2_{V_{i}^{i}}^{\Gamma_{i}}$, then $Y_{n}$ is a $Q$-factor by the Attaching Theorem.

Proposition 3.3. If $\Gamma$ is a finite, connected, acyclic graph and $V$ is any subset (possibly empty) of the vertices of $\Gamma$, then $2_{V}^{\Gamma}$ is a Q-factor.

Proof. (By induction on the number of edges in $\Gamma$.) If $\Gamma$ is degenerate (no edges), this is clear, and if $\Gamma$ has only one edge, this is shown in [10]. Now suppose that $\Gamma$ has more than one edge and that the proposition is true for graphs with fewer edges than $\Gamma$. Adopt the notation of this section but allow $w$ to belong to $V$. If 
$w \in V$, then the mapping $\prod_{i=1}^{n} 2_{V_{i}}^{\Gamma_{i}} \rightarrow 2_{V}^{\Gamma}$ given by $\left(A_{1}, \cdots, A_{n}\right) \rightarrow$ $A_{1} \cup \cdots \cup A_{n}$ is a homeomorphism and since each of the $2_{V_{i}^{i}}^{\Gamma_{i}}$ is a $Q$ factor by the inductive hypothesis, $2_{V}^{\Gamma}$ is also a $Q$-factor.

If $w \notin V$, then by the above we have that $2_{W}^{\Gamma}$ is a $Q$-factor and hence by Lemma 3.2, $Y_{n}$ is a $Q$-factor. For $k=1, \cdots, n-1$, let $Y_{k}$ be the subset of $2_{V}^{r}$ composed of those members which meet at least $k$ of the $\Gamma_{i}$ 's. If $Y_{k+1} \neq 2_{V}^{\Gamma}$, let $\sigma_{1}, \cdots, \sigma_{p}$ be the subsets of $\{1, \cdots, n\}$ with exactly $k$ members which contain $\left\{i: 1 \leqq i \leqq n, V_{i} \neq\right.$ $\varnothing\}$, and let

$$
X_{\sigma_{j}}=\bigcup_{i \in \sigma_{j}}\left(2_{W_{i}}^{\Gamma_{i}} \times \prod_{m \in \sigma_{j} \backslash\{i\}} 2_{V_{m}^{m}}^{\Gamma_{m}}\right) .
$$

Then exactly as in the proof of Lemma 3.1, each $X_{o_{j}}$ is a $Q$-factor and a $Z$-set in $\Pi_{i \in \sigma_{j}} 2_{V_{i}^{i}}^{\Gamma_{i}}$. For $i=1, \cdots, p$, let $Y_{k, 2}$ be the subset of $2_{V}^{\Gamma}$, composed of those members that are contained in $\mathrm{U}_{j \in \sigma_{i}} \Gamma_{j}$ and which meet each $\Gamma_{i}, j \in \sigma_{i}$; let $Y_{k}^{j}=\left(\bigcup_{j=1}^{i} Y_{k, j}\right) \cup Y_{k+1}$, and let $Y_{k}^{o}$ denote $Y_{k+1}$. Then $Y_{k}=Y_{k}^{p}$ and $f_{k, i}:\left(\prod_{j \in \sigma_{i}} 2_{V_{j}^{j}}^{j}, X_{\sigma_{i}}\right) \rightarrow\left(Y_{k}^{i}, Y_{k}^{i-1}\right)$ defined by $f_{k, i}\left(A_{1}, \cdots, A_{k}\right)=A_{1} \cup \cdots \cup A_{k}$ is a relative homeomorphism and hence $Y_{k}^{i} \approx \prod_{j \in \sigma_{i}} 2_{v j}^{\Gamma_{j}^{j}} \bigcup_{g} Y_{k}^{i-1}$, where $g=f_{k, i} \mid X_{\sigma_{i}}$. Thus, by induction we have that $Y_{k}=Y_{k}^{p}$ is a $Q$-factor if $Y_{k+1}=Y_{k}^{0}$ is one. Thus, since $Y_{n}$ is a $Q$-factor we have by induction that $Y_{1}=2_{V}^{\Gamma}$ is a $Q$-factor.

THEOREM 3.4. If $\Gamma$ is a finite, connected graph and $V$ is any subset (possibly empty) of the vertices of $\Gamma$, then $2_{V}^{\Gamma} i s$ a $Q$-factor.

Proof. As this is a topological result, new vertices may be introduced in $\Gamma$ at will and therefore, one may assume without loss of generality that for some connected, acyclic graph $\Gamma_{0}$ and some collection $v_{1}, w_{1}, \cdots, v_{n}, w_{n}$ of free vertices of $\Gamma_{0}$, that $\Gamma=\Gamma_{0} / R$ where $R$ is the equivalence relation on $\Gamma_{0}$ generated by $v_{i} R w_{i}$ for $i=1, \cdots, n$. For $1 \leqq k \leqq n$, let $R_{k}$ be the equivalence relation on $\Gamma_{0}$ generated by $v_{i} R w_{i}$ for $i=1, \cdots, k$, and let $\Gamma_{k}=\Gamma_{0} / R_{k}$. Since $R_{k-1} \subset R_{k}$, we have a natural map $\varphi_{k}: \Gamma_{k-1} \rightarrow \Gamma_{k}$ induced by the identity map on $\Gamma_{0}$.

The theorem is true for $\Gamma_{0}$ by Proposition 3.3. Suppose the theorem is true for $\Gamma_{k-1}$, let $X$ be any subset of the vertices of $\Gamma_{k}$ and let $X^{\prime}=\varphi_{k}^{-1}(X)$. Let $f_{k}: 2_{X^{\prime}}^{\Gamma_{k-1}} \rightarrow 2_{X}^{\Gamma_{k}}$ be the map induced by $\varphi_{k}$ and observe that $f_{k}$ carries $2_{X^{\prime} \cup\left\{v_{k}, w_{k}\right\}}^{\Gamma^{k}-1}$ homeomorphically onto $2_{\left.X \cup \varphi_{k}\left(1 v_{k}, w_{k}\right)\right\}}^{\Gamma_{k}}$. Thus, if $\varphi_{k}\left(\left\{v_{k}, w_{k}\right\}\right) \in X$, then $2_{X}^{\Gamma_{k}}$ is a $Q$-factor. If $\varphi_{k}\left(\left\{v_{k}, w_{k}\right\}\right) \notin X$, let $Y_{1}=X^{\prime} \cup\left\{v_{k}\right\}, \quad Y_{2}=X^{\prime} \cup\left\{w_{k}\right\}$, and $Y_{3}=X^{\prime} \cup\left\{v_{k}, w_{k}\right\}$. Then $2_{X^{\prime}}^{\Gamma_{k-1}}, 2_{Y_{i}}^{\Gamma_{k-1}}, i=1,2,3$, and $2_{\varphi_{k}}^{\Gamma_{k}^{k}\left(Y_{3}\right)}$ are $Q$-factors and $2_{Y_{3}^{k-1}}^{\Gamma_{k-1}}=2_{Y_{1}}^{\Gamma_{k-1}} \cap 2_{Y_{2}}^{\Gamma_{k-1}}$. Moreover, since $v_{k}$ and $w_{k}$ are free vertices, $2_{Y}^{\Gamma_{k}^{k-1}}$ is a $Z$-set in each of them and thus by the First Sum Theorem $2_{Y_{1}}^{\Gamma-1} \cup 2_{Y_{2}-1}^{\Gamma_{k-1}}$ is a $Q$ factor. Also, since each of $2_{Y_{i}^{k-1}}^{\Gamma_{k-1}}, i=1,2$, is a $Z$-set in $2_{X^{\prime}}^{\Gamma_{k-1}}$, their 
union is also a $Z$-set by $2.2(\mathrm{~b})$. Moreover, $f_{k}:\left(2_{X^{\prime}}^{\Gamma_{k}-1}, 2_{Y_{1}^{k}}^{\Gamma_{k}-1} \cup 2_{Y_{2}}^{\Gamma_{k-1}}\right) \rightarrow$ $\left(2_{X}^{\Gamma_{k}}, 2_{\varphi_{k}\left(Y_{3}\right)}^{\Gamma_{k}}\right)$ is a relative homeomorphism and hence $2_{X}^{\Gamma_{k}} \approx 2_{X^{\prime}}^{\Gamma_{k}^{1}-1} \bigcup_{g_{k}} 2_{\varphi_{k}^{k}\left(Y_{3}\right)}^{\Gamma_{k}}$ where $g_{k}=f_{k} \mid 2_{Y_{1}}^{\Gamma_{k-1}} \cup 2_{Y_{2}}^{\Gamma_{k-1}}$, and thus by the Attaching Theorem $2_{X}^{\Gamma_{k}}$ is a $Q$-factor and the theorem follows.

4. $2^{\Gamma}$ is a Hilbert cube. In this section we verify the last three conditions of the Compactification Theorem.

Lemma 4.1. If $\Gamma$ is a finite, connected graph and $V$ is any set of vertices (possibly empty) of $\Gamma$, then $C_{V}(\Gamma)$ is a $Q$-factor.

Proof. First we show that $C_{V}(\Gamma)$ is contractible. Let $\Gamma$ be endowed with a convex metric, i.e., one for which there always exists a point half way between any two given points. Then the function $F: C_{V}(\Gamma) \times I \rightarrow C_{V}(\Gamma)$ defined by $F(A, t)$ is equal to the closed t $\delta$-neighborhood of $A$ in $\Gamma$, where $\delta$ is the diameter of $\Gamma$, is a contraction of $C_{V}(\Gamma)$ to the point $\Gamma \in C_{V}(\Gamma)$.

Next, in [8], R. Duda proves that $C(\Gamma)$ is a polyhedron and since it is contractible we have by [11] that $C(\Gamma)$ is a $Q$-factor. If $V \neq$ $\varnothing$, then $C_{V}(\Gamma)$ is geometrically easier to classify than $C(\Gamma)$ and although it was not specifically dealt with in [8], it is a subpolyhedron of $C(\Gamma)$, and since it is contractible, it is a $Q$-factor. For a considerably more general result see [6].

LEMMA 4.2. If $\Gamma$ is a finite, connected, nondegenerate graph, $w$ is a vertex of $\Gamma$, and $V$ is a collection (possibly empty) of vertices of $\Gamma$, then $C_{V \cup\{w\}}(\Gamma)$ is a $Z$-set in $2_{V}^{\Gamma}$.

Proof. We will first prove the result for the case that $w \in V$ by constructing for each $\varepsilon>0$ a map $f: 2_{V}^{\Gamma} \rightarrow 2_{V}^{\Gamma} \backslash C_{V}(\Gamma)$ that is within $\varepsilon$ of the identity. Let $w_{i}, i=1, \cdots, n$, be the vertices of $\Gamma$ which are joined to $w$ by edges $E_{i}=\left[w, w_{i}\right]$ and assume, for the metric on $\Gamma$, that each $E_{i}$ is isometric with $[0,1]$ so that for each $0<\varepsilon \leqq$ 1 the open $\varepsilon$-ball about $w, U(w, \varepsilon)$, is precisely the set $\{(1-t) w+$ $\left.t w_{i}: 0 \leqq t<\varepsilon, i=1, \cdots, n\right\}$. Let $V(w, \varepsilon)$ be the closure in $\Gamma$ of $U(w, \varepsilon)$ and let $\operatorname{Bd} U(w, \varepsilon)=V(w, \varepsilon) \backslash U(w, \varepsilon)$. For a fixed $0<\varepsilon<1$, and for $A \in 2_{V}^{\Gamma}$, let

$$
f(A)=[A \backslash U(w, \varepsilon / 2)] \cup\{w\} \cup \operatorname{Bd} U(w, \varepsilon / 2) .
$$

It is clear that $[A \backslash U(w, \varepsilon / 2)] \cup\{w\} \in 2_{V}^{\Gamma} \backslash C_{V}(\Gamma)$ but this assignment of $A$ would not be continuous basically for the reason that one may have two points $x \in U(w, \varepsilon / 2)$ and $y \notin U(w, \varepsilon / 2)$ that are very close together. Including the set $\operatorname{Bd} U(w, \varepsilon / 2)$ in the image under $f$ of $A$ 
establishes the continuity of $f$, which is within $\varepsilon$ of the identity map because in $2_{V}^{r}$ the distance between $\{w\}$ and $\mathrm{Bd} U(w, \varepsilon / 2)$ is $\varepsilon / 2<\varepsilon$. Thus, since $f$ is continuous and the image of $f$ misses $C_{V}(\Gamma), C_{V}(\Gamma)$ is a $Z$-set in $2_{V}^{r}$.

We will now modify these techniques to prove the theorem in the case $w \notin V$ : Let $W=V \cup\{w\}$. If the above map $f$ were defined on $2_{V}^{r}$ it would not be within $\varepsilon$ of the identity, as is seen by comparing $f(A)$ and $A$ for sets $A$ with no points close to $w$. Since our main technique of mapping $2_{V}^{\Gamma}$ off $C_{W}(\Gamma)$ is to delete an open set about $w$, we will phase out this process so that we will be deleting open sets about $w$ only from those members of $2_{V}^{r}$ that contain points close to $w$.

For $0 \leqq a \leqq 1$ we denote the point $(1-a) w+a w_{i} \in\left[w, w_{i}\right]$ simply by $[a]_{i}$. For $A \in 2_{V}^{\Gamma}$, let $a_{i} \in[0,1]$ be the number such that $\left[a_{i}\right]_{i}$ is the point of $A \cap E_{i}$ nearest to $w$, if $A \cap E_{i} \neq \varnothing$. If $0 \leqq a_{i} \leqq \varepsilon$, let $a_{i}^{\prime}=\max \left\{0,2 a_{i}-\varepsilon\right\}$ observing that if $0 \leqq a_{i} \leqq \varepsilon / 2$, then $a_{i}^{\prime}=0$; and if $a_{i}=\varepsilon$, then $a_{i}^{\prime}=a_{i}$. For $A \in 2_{V}^{r}$, let

$$
f(A)=\left\{\begin{array}{r}
A \cup\left\{\left[a_{i}^{\prime}\right]_{i}: 1 \leqq i \leqq n, 0 \leqq a_{i} \leqq \varepsilon\right\}, \quad \text { if } \delta \geqq \varepsilon / 2 \\
A \cup\left\{\left[(2 \delta / \varepsilon) a_{i}^{\prime}+(1-2 \delta / \varepsilon) a_{i}\right]_{i}: 1 \leqq i \leqq n, 0 \leqq a_{i} \leqq \varepsilon\right\} \\
\text { if } 0 \leqq \delta \leqq \varepsilon / 2
\end{array}\right.
$$

where $\delta=\delta(A)=D\left(A, 2_{w}^{\Gamma}\right)$, which in this case is the minimum distance between points of $A$ and $w$. Then $f$ is a well-defined function since it is uniquely defined for elements $A \in 2_{V}^{\Gamma}$, where $\delta=\varepsilon / 2$. Also, $f$ is phased back to the identity at $\delta=0$, that is, if $\delta(A)=0$, then $f(A)=A$; and this establishes the continuity of $f$. Also observe that if $\delta(A)=\varepsilon / 2$, then $w \in f(A)$ and if $\delta(A) \geqq \varepsilon$, then $f(A)=A$. Let $\alpha(A)=\max \{0, \varepsilon / 2-\delta(A)\}$ and define $g$ on $f\left(2_{V}^{r}\right)$ by

$$
g f(A)= \begin{cases}{[f(A) \backslash U(w, \alpha(A))] \cup \operatorname{Bd} U(w, \alpha(A))} & \text { if } \delta(A)<\varepsilon / 2 \\ f(A) & \text { if } \delta(A) \geqq \varepsilon / 2 .\end{cases}
$$

The continuity of $g$ follows since $\alpha$ is continuous and since for $A \in 2_{V}^{r}$ where $\delta(A)$ is less than $\varepsilon / 2$ but close to $\varepsilon / 2$, then $\operatorname{Bd} U(w, \alpha(A))$ is close to $\{w\}$, and hence $g f(A)$ is close to $f(A)$. Furthermore, the composition $g f: 2_{V}^{\Gamma} \rightarrow 2_{V}^{\Gamma}$ is within $\varepsilon$ of the identity and $g f\left(2_{V}^{\Gamma}\right) \cap C_{W}(\Gamma)=$ $\varnothing$ and thus, $C_{W}(\Gamma)$ is a $Z$-set in $2_{V}^{\Gamma}$.

The next lemma will be the inductive step for the main theorem of this section. Let $L_{1}, \cdots, L_{m}$ be a finite collection of finite, connected graphs, let $W$ be a collection of vertices from $\bigcup_{i=1}^{m} L_{i}$ where $W$ contains at least one vertex of each $L_{i}$, and let $K=$ $\left(\bigcup_{i=1}^{m} L_{i}\right) / W$ be the quotient space obtained by taking the disjoint union of the $L_{i}$ and identifying all the vertices in $W$. Let $p: \bigcup_{i=1}^{m} L_{i} \rightarrow$ $K$ be the quotient map and let $w=p(W)$. 
LEMMA 4.3. If each $2_{V_{i}}^{L i}$ is a Hilbert cube for each collection $V_{i}$ (possibly empty) of vertices of $L_{i}$, then $2_{V}^{K}$ is a Hilbert cube for each set of vertices $V$ (possibly empty) of $K$.

Proof. To apply the Compactification Theorem, we have that $2_{V}^{K}$ is a $Q$-factor by $3.4, C_{W}(K)$ is a $Q$-factor by 4.1 where $W=V \cup$ $\{w\}$, and $C_{W}(K)$ is a $Z$-set in $2_{V}^{K}$, by 4.2 . It remains to be shown that $2_{V}^{K} \backslash C_{W}(K)$ is a $Q$-manifold.

If $A \in 2_{V}^{K} \backslash C_{W}(K)$, then $A$ has a component missing $w$. If $A$ is connected, then it has an open neighborhood $U$ in $2_{V}^{K}$ homeomorphic to an open set of $2_{V_{i}}^{L_{i}}$, for some $i$ and some collection $V_{i}$ of vertices of $L_{i}$. Since $2_{V_{i}}^{L_{i}}$ is by hypothesis a Hilbert cube, $U$ is homeomorphic to an open subset of the Hilbert cube. If $A$ is not connected, then it has a separation into two disjoint closed nonempty subsets $A_{1}$ and $A_{2}$ such that $A=A_{1} \cup A_{2}$. Assuming that $w \notin A_{2}$, let $U_{1}$ and $U_{2}$ be disjoint open sets of $K$ containing $A_{1}$ and $A_{2}$, respectively. Now, for some $i_{1}, \cdots, i_{k}, 1 \leqq k \leqq m, A_{2}$ has an open neighborhood $W_{2}$ in $2_{A_{2} \cap V}^{K}$ consisting of sets lying entirely within $U_{2}$, which is homeomorphic to a product $U_{21} \times U_{22} \times \cdots \times U_{2 k}$ of open sets of the Hilbert cubes $2_{V j}^{L_{j}}, j=i_{1}, \cdots, i_{k}$ where $V_{j}=L_{j} \cap p^{-1}\left(A_{2} \cap V\right)$. On the other hand, the set $W_{1}=\left\{B \in 2_{V^{\prime}}^{K}: B \subset U_{1}\right\}$, where $V^{\prime}=V \cap A_{1}$, is an open neighborhood of $A_{1}$ in $2_{V^{\prime}}^{K}$ which is by 3.4 a $Q$-factor. Now $U=\left\{B \cup C: B \in W_{1}, C \in W_{2}\right\}$ is an open neighborhood of $A$ in $2_{V}^{K}$ which is homeomorphic to $W_{1} \times W_{2}$ and hence, to an open subset of the Hilbert cube $2_{V^{\prime}}^{K} \times \Pi\left\{2_{V_{j}^{j}}^{L}: j=i_{1}, \cdots, i_{k}\right\}$. Therefore, $2_{V}^{K} \backslash C_{W}(K)$ is a $Q$ manifold and the proof is complete.

THEOREM 4.4. If $\Gamma$ is a nondegenerate, finite, connected graph and $V$ is any set (possibly empty) of vertices of $\Gamma$, then $2_{V}^{\Gamma}$ is a Hilbert cube.

Proof. Let $\mathscr{G}$ be the class of all nondegenerate, finite, connected graphs. For each $K \in \mathscr{G}$, let $V(K)$ be the number of vertices of $K, E(K)$ the number of edges of $K$, and $R(K)=E(K)-V(K)+$ 1. $\left(R(K)\right.$ is the rank of the first homology group $H_{1}(K)$; it is also $E(K)-E(L)$ for each maximal acyclic subgraph $L$ of $K$.) Let $\mathscr{G}_{i}$ be the class of all members $K$ of $\mathscr{G}$ for which $R(K)=i$, and let $\mathscr{G}_{i j}$ be the subclass of $\mathscr{G}_{i}$ composed of all members $K$ of $\mathscr{G}_{i}$ with $E(K)=j$.

The theorem holds for $\mathscr{G}_{01}$, being the main results of [9] and [10]. Specifically, $2^{I}, 2_{0}^{I}, 2_{1}^{I}$, and $2_{01}^{I}$ are all Hilbert cubes. Now fix $(i, j) \neq(0,1)$ and suppose that the theorem holds for each $\mathscr{G}_{i^{\prime} j^{\prime}}$, with $i^{\prime}<i$ or $i^{\prime}=i$ and $j^{\prime}<j$. 
Let $K \in \mathscr{G}_{i j}$ and let $V$ be a set of vertices (possibly empty) of $K$ and let $w$ be a vertex of $K$ which is not a free vertex of $K$. Construct a new complex $K^{\prime}$ by "splitting" $K$ at $w$. That is, let $v_{1}, \cdots, v_{n}$ be the vertices of $K$ which are joined to $w$ by edges $\left[w, v_{i}\right]$ of $K$ and let $w_{1}, \cdots, w_{n}$ be abstract vertices not in $K$. Then $K^{\prime}=\left(K \backslash \bigcup_{i=1}^{n}\left[w, v_{i}\right)\right) \cup \bigcup_{i=1}^{n}\left[w_{i}, v_{i}\right]$ and $K^{\prime}$ has as vertices all vertices of $K$ except $w$ together with $w_{1}, \cdots, w_{n}$ and has as edges all edges of $K$ which do not contain $w$ together with the new edges $\left[w_{i}, v_{i}\right]$, $i=1, \cdots, n$. Now, if $w$ separates $K$, each component $L$ of $K^{\prime}$ has $E(L)<E(K)$ and $R(L) \leqq R(K)$, while if $w$ does not separate $K$, then $K^{\prime} \in \mathscr{G}$ and $R\left(K^{\prime}\right)<R(K)$. Thus, by the induction hypothesis, each component of $K^{\prime}$ satisfies the theorem and hence by Lemma 4.3, $2_{V}^{K}$ is a Hilbert cube and thus by induction the theorem is proved.

5. $2^{D}$ and $C(D)$ for local dendrons $D$. In this section we generalize the theorems to each dendron, that is, a Peano space which contains no simple closed curve, and to each local dendron, that is, a Peano space such that each point has a closed neighborhood which is a dendron. In particular, each dendron is a local dendron. We can express (see [13]) each dendron $D$ as the limit of an inverse sequence $\left(T_{n}, r_{n}\right)$, $\lim \left(T_{n}, r_{n}\right)$, where $T_{1}$ is an arc and for each $n \geqq 1$, $T_{n+1}$ is the union of $T_{n}$ and an arc $\left[a_{n}, b_{n}\right]$ where $T_{n} \cap\left[a_{n}, b_{n}\right]=\left\{a_{n}\right\}$, and where $r_{n}: T_{n+1} \rightarrow T_{n}$ is the retraction which collapses $\left[a_{n}, b_{n}\right]$ to $a_{n}$. The inverse sequence $\left(T_{n}, r_{n}\right)$ induces the inverse sequence $\left(2^{T}, r_{n}^{*}\right)$ where $r_{n}^{*}: 2^{T_{n+1}} \rightarrow 2^{T}$ is defined by $r_{n}^{*}(A)=r_{n}(A)$. Then $2^{D}$ is homeomorphic to $\lim \left(2^{T}, r_{n}^{*}\right)$.

The corresponding inverse limit representation for local dendrons is the same except that $T_{1}$ is allowed to be a finite, connected graph. We argue this as follows. For a local dendron $D$ there exists an $\varepsilon>0$ such that each closed connected subset of $D$ with diameter less than $\varepsilon$ is a dendron. Cover $D$ with a finite collection of closed connected neighborhoods $\left\{D_{i}\right\}$ with diameter less than $\varepsilon / 2$. The pairwise intersections of the $D_{i}$ are connected. In each nonempty intersection of elements of the $\left\{D_{i}\right\}$ pick a point and then in each $D_{i}$ construct a tree connecting each of the selected points contained in that $D_{r}$. Then the union of these trees will be a finite connected graph, a candidate for $T_{1}$ in the above inverse limit presentation. Now we can add the remaining stickers to the trees in the prescribed manner to obtain the local dendron $D$ as the $\lim \left(T_{n}, r_{n}\right)$. Such an inverse limit for a local dendron $D$ will be called a standard inverse limit representation for $D$. Also, for a given finite subset $V$ of $D$ we can easily construct $T_{1}$ to contain $V$ by including it in the set of points picked in the intersections of the $D_{i}$. We will need the next result. 
Theorem 5.1. Morton Brown [3]. Let $S=\lim \left(X_{n}, f_{n}\right)$, where the $X_{n}$ are all homeomorphic to a given compact metric space $X$ and each $f_{n}$ is a near-homeomorphism. Then $S$ is homeomorphic to $X$.

Lemma 5.2. If $f: Q \rightarrow Q$ is a map that stabilizes to a nearhomeomorphism, then $f$ is a near-homeomorphism.

Proof. Define $\alpha_{n}: Q \times Q \rightarrow Q$ by $\alpha_{n}\left(\left(x_{1}, x_{2}, \cdots\right),\left(y_{1}, y_{2}, \cdots\right)\right)=$ $\left(x_{1}, \cdots, x_{n}, y_{1}, x_{n+1}, y_{2}, x_{n+2}, y_{3}, \cdots\right)$. Then each $\alpha_{n}$ is a homeomorphism and hence each $\alpha_{n} \circ(f \times i d) \circ \alpha_{n}^{-1}$ is a near-homeomorphism since $f \times i d$ is one by assumption. Furthermore, $d\left(f, \alpha_{n} \circ(f \times i d) \circ \alpha_{n}^{-1}\right) \rightarrow 0$ as $n \rightarrow$ $\infty$ and hence $f$ is a uniform limit of near-homeomorphisms and thus is a near-homeomorphism.

THeOREM 5.3. If $D$ is a nondegenerate local dendron and $V$ is any finite subset (possibly empty) of $D$, then $2_{V}^{D}$ is a Hilbert cube.

Proof. We follow the proof of [Theorem 2, 13] which states a corresponding result for $C(D)$. Choose a standard inverse limit representation for $D$ where $V \subset T_{1}$. Let $r_{n}^{\prime}: 2_{V \cup\left\{b_{n}\right\}}^{T} \rightarrow 2_{V}^{T}{ }^{n}$ be the restriction of the map $r_{n}^{*}$, let $M_{r_{n}^{\prime}}$ be the mapping cylinder of $r_{n}^{\prime}$, and let $c_{n}: M_{r_{n}^{\prime}} \rightarrow 2_{V}^{T}$ be the natural projection defined by $c_{n}([A, t])=r_{n}^{\prime}(A)$. Since $2_{V \cup\left\{b_{n}\right\}}^{T n+1}$ and $2_{V}^{T}$ are $Q$-factors by 3.4 , it follows by the Mapping Cylinder Theorem that $c_{n}$ stabilizes to a near-homeomorphism. We will show below that $M_{r_{n}^{\prime}}$ is homeomorphic to $2_{V}^{T n+1}$ in such a way that the projection map $c_{n}$ is topologically equivalent to $r_{n}^{*}$. Thus, since each of $2_{V}^{T}$ and $2_{V}^{T} n+1$ is a Hilbert cube, we have by 5.2 that $c_{n}$ is a near-homeomorphism and hence so is $r_{n}^{*}$. The proof that $2_{V}^{D} \approx Q$ will then be complete by 5.1 since $2_{V}^{D}$ is homeomorphic to an inverse limit of Hilbert cubes $2_{V}^{T} n$ where the bonding maps are nearhomeomorphisms. We now verify the above stated fact about $M_{r_{n}^{\prime}}$. Define $g_{n}: 2_{V}^{T} n \rightarrow 1+M_{r_{n}^{\prime}}$ as follows where we parametrize $\left[a_{n}, b_{n}\right]$ to be order isomorphic with $[0,1]$ and let $\sup \left(A \cap\left[a_{n}, b_{n}\right]\right)=d$ if it exists. Let

$$
g_{n}(A)= \begin{cases}{[A],} & \text { if } A \cap\left(a_{n}, b_{n}\right]=\varnothing \\ {\left[\left(A \cap T_{n}\right) \cup\left(1 / d\left(A \cap\left[a_{n}, b_{n}\right]\right), d\right)\right],} & \text { if } A \cap\left(a_{n}, b_{n}\right] \neq \varnothing .\end{cases}
$$

Then $g_{n}$ is a homeomorphism so that the following diagram is

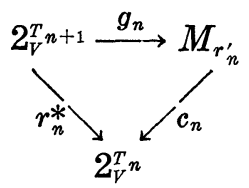


commutative and this completes the proof.

In [13], it is proved that the subcontinua $C(D)$ of a dendron $D$ form a $Q$-factor which is a Hilbert cube if and only if the branch points of $D$ are dense. We will extend this result to local dendrons $D$ and to spaces $C_{V}(D)$ where $V$ is a finite subset of $D$.

Lemma 5.4. For each local dendron $D$ and each finite subset $V$ (possibly empty) of $D, C_{V}(D)$ is a $Q$-factor.

Proof. Choose a standard inverse limit representation, $\lim \left(T_{n}, r_{n}\right)$, for $D$ where $V \subset T_{1}$. Then $C_{V}(D) \approx \lim \left(C_{V}\left(T_{n}\right), r_{n}^{*}\right)$. As in the proof of Theorem 5.3 the space $C_{V}\left(T_{n+1}\right)$ is naturally homeomorphic to the mapping cylinder $M_{r_{n}^{\prime}}$ where $r_{n}^{\prime}: C_{V \cup\left\{b_{n}\right\}}\left(T_{n+1}\right) \rightarrow C_{V}\left(T_{n}\right)$ is the restriction of $r_{n}^{*}$. Furthermore, the map $r_{n}^{*}$ is topologically equivalent to the natural projection $c_{n}: M_{r_{n}^{\prime}} \rightarrow C_{V}\left(T_{n}\right)$ which stabilizes to a nearhomeomorphism. Since each space $C_{V}\left(T_{n}\right)$ is a $Q$-factor by Lemma 4.1 and since each bounding map $r_{n}^{*}$ stabilizes to a near-homeomorphism, then $C_{V}(D) \approx \lim \left(C_{V}\left(T_{n}\right), r_{n}^{*}\right)$ is a $Q$-factor and the proof is complete.

To prove that $C_{V}(D)$ is a Hilbert cube if the branch points of $D$ are dense, we will need Lemmas 4.1 and 5.4 together with the next two lemmas to satisfy the hypothesis of the Compactification Theorem where $X=C_{V}(D)$ and $A=C_{V}\left(T_{1}\right)$.

LEMMA 5.5. Let $D$ be a local dendron with a dense set of branch points, let $V$ be a finite subset (possibly empty) of $D$, and let $\lim \left(T_{n}, r_{n}\right)$ be a standard inverse limit representation for $D$ where $V \subset T_{1}$. Then $C_{V}\left(T_{1}\right)$ is a $Z$-set in $C_{V}(D)$.

Proof. A local dendron admits a convex metric. Using a convex metric on $D$, for sufficiently small $\varepsilon>0$, the map $f$ on $C_{V}(D)$ defined by setting $f(A)$ equal to the closed $\varepsilon$-neighborhood of $A$ in $D$ is a map from $C_{V}(D)$ into itself where $d(f, i d)<\varepsilon$. Since the branch points of $D$ are dense, we also have that $f: C_{V}(D) \rightarrow C_{V}(D) \backslash C_{V}\left(T_{1}\right)$ and hence $C_{V}\left(T_{1}\right)$ is a $Z$-set in $C_{V}(D)$.

Lemma 5.6. If $D, V$, and $\lim \left(T_{n}, r_{n}\right)$ are as above, then $C_{V}(D) \backslash C_{V}\left(T_{1}\right)$ is a Q-manifold.

Proof. Let $A \in C_{V}(D) \backslash C_{V}\left(T_{1}\right)$. It is sufficient, since $C_{V}(D) \backslash C_{V}\left(T_{1}\right)$ is open in $C_{V}(D)$, to show that $A$ has an open neighborhood in $C_{V}(D)$ that is homeomorphic to an open subset of the Hilbert cube. If $A \cap T_{1}$ is either empty or a single point, then $V$ is either empty or is a single point and there exists an open set $U$ in $D$ containing $A$ and a dendron $D_{1}$ such that $A \subset U \subset D_{1} \subset D$. If $W$ is the set of all 
elements of $C_{V}(D)$ contained in $U$, then $W$ is an open neighborhood of $A$ in $C_{V}(D)$ and is an open subset of $C_{V}\left(D_{1}\right)$ which is a Hilbert cube by an obvious modification of West's proof [13] that $C\left(D_{1}\right)$ is a Hilbert cube.

If $A \cap T_{1}$ is nondegenerate, let $E$ be the closure of some component of $D \backslash T_{1}$ that contains some points of $A$ and let $F$ be the closure of $D \backslash E$. Then $E$ is a dendron and $F$ is a local dendron containing $T_{1}$ and each has a dense set of branch points and $E \cap F$ is one point, say $q$. Then $C_{q}(E)$ is a Hilbert cube by modifying West's argument and $C_{W}(F)$, where $W=V \cup\{q\}$, is a $Q$-factor by Lemma 5.4 and hence $C_{q}(E) \times C_{W}(F)$ is a Hilbert cube. The map $\alpha: C_{q}(E) \times$ $C_{W}(F) \rightarrow C_{V}(D)$ defined by $\alpha(A, B)=A \cup B$ is an embedding into $C_{V}(D)$ where the image of $\alpha$ is a closed neighborhood (not a small one) of $A$ and thus $C_{V}(D) \backslash C_{V}\left(T_{1}\right)$ is a $Q$-manifold.

Theorem 5.7. If $D$ is a local dendron and $V$ is a finite subset (possibly empty) of $D$, then $C_{V}(D)$ is a Q-factor, and furthermore if the branch points of $D$ are dense, then $C_{V}(D)$ is a Hilbert cube.

Proof. The first part of the theorem is Lemma 5.4 and the second part follows from applying Lemmas 4.1 and 5.4-5.6 to the Compactification Theorem and observing that $D$ admits a standard inverse limit representation $\lim \left(T_{n}, r_{n}\right)$ where $V \subset T_{1}$.

\section{REFERENCES}

1. R. D. Anderson, On topological infinite deficiency, Mich. Math. J., 14 (1967), 365-383.

2. C. Bessaga and A. Pelczynski, Estimated extension theorem, homogeneous collections and skeletons, and their applications to topological classification of linear metric spaces, Fund. Math., 69 (1970), 153-190.

3. M. Brown, Some applications of an approximation theorem for inverse limits, Proc. Amer. Math. Soc., (1960), 478-483.

4. T. A. Chapman, Notes on Hilbert cube manifolds, (Mimeographed notes, University of Kentucky).

5. D. W. Curtis and R. M. Schori, $2^{X}$ and $C(X)$ are homeomorphic to the Hilbert cube, Bull. Amer. Math. Soc., (to appear).

6. - Hyperspaces of Peano continua are Hilbert cubes, (in preparation).

7. J. Eells and N. H. Kuiper, Homotopy negligible subsets of infinite-dimensional manifolds, Compositio Math., 21 (1969), 155-161.

8. R. Duda, On the hyperspace of subcontinua of a finite graph I, Fund. Math., 62 (1968), 265-286.

9. R. M. Schori and J. E. West, $2^{I}$ is homeomorphic to the Hilbert cube, Bull. Amer. Math. Soc., 78 (1972), 402-406.

10. - The hyperspace of closed subsets of the closed unit interval is a Hilbert cube, Trans. Amer. Math. Soc., (to appear).

11. J. E. West, Infinite products which are Hilbert cubes, Trans. Amer. Math. Soc., 150 (1970), 1-25.

12. - Mapping cylinders of Hilbert cube factors, General Topology, 1 (1971), 111-125. 
13. J. E. West, The subcontinua of a dendron form a Hilbert cube factor, Proc. Amer. Math. Soc., 36 (1972), 603-608.

14. - Sums of Hilbert cube factors, Pacific J. Math., (to appear).

15. - Mapping cylinders of Hilbert cube factors II, General Topology, 1 (1971), 111-125.

16. M. Wojdyslawski, Sur la contractilite des hyperspaces de continus localement connexes, Fund. Math., 30 (1938), 247-252.

Received June 15, 1973. Research supported in part by NSF Grants GP-34635X and GP-16862.

LoUisiana State University

AND

CoRnell UNIVERSITY 



\section{PACIFIC JOURNAL OF MATHEMATICS}

\section{EDITORS}

RICHARD ARENS (Managing Editor)

University of California

Los Angeles, California 90024

R. A. Beaumont

University of Washington

Seattle, Washington 98105
J. DugundJI

Department of Mathematics

University of Southern California

Los Angeles, California 90007

D. Gilbarg and J. Milgram

Stanford University

Stanford, California 94305

\section{ASSOCIATE EDITORS}

E. F. BECKENBACH

B. H. NeUMANN

F. WOLF

K. YosHIDA

\section{SUPPORTING INSTITUTIONS}

UNIVERSITY OF BRITISH COLUMBIA

CALIFORNIA INSTITUTE OF TECHNOLOGY

UNIVERSITY OF CALIFORNIA

MONTANA STATE UNIVERSITY

UNIVERSITY OF NEVADA

NEW MEXICO STATE UNIVERSITY

OREGON STATE UNIVERSITY

UNIVERSITY OF OREGON

OSAKA UNIVERSITY
UNIVERSITY OF SOUTHERN CALIFORNIA

STANFORD UNIVERSITY

UNIVERSITY OF TOKYO

UNIVERSITY OF UTAH

WASHINGTON STATE UNIVERSITY

UNIVERSITY OF WASHINGTON

$\stackrel{*}{*} \stackrel{*}{*} \stackrel{*}{*}$ AMERICAN MATHEMATICAL SOCIETY




\section{Pacific Journal of Mathematics}

\section{Vol. 53, No. $1 \quad$ March, 1974}

Martin Bartelt, Strongly unique best approximates to a function on a set, and a finite

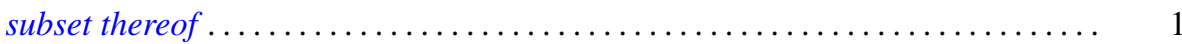

S. J. Bernau, Theorems of Korovkin type for $L_{p}$-spaces $\ldots \ldots \ldots \ldots \ldots \ldots \ldots \ldots \ldots$

S. J. Bernau and Howard E. Lacey, The range of a contractive projection on an

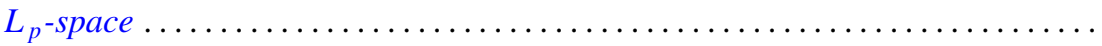

Marilyn Breen, Decomposition theorems for 3-convex subsets of the plane ......... Ronald Elroy Bruck, Jr., A common fixed point theorem for a commuting family of

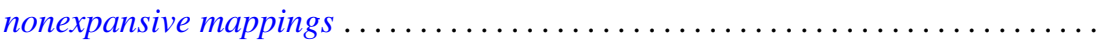

Aiden A. Bruen and J. C. Fisher, Blocking sets and complete $k$-arcs . . . . . . . 73

R. Creighton Buck, Approximation properties of vector valued functions . ......... 85

Mary Rodriguez Embry and Marvin Rosenblum, Spectra, tensor products, and

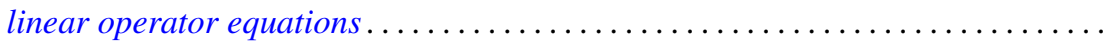

Edward William Formanek, Maximal quotient rings of group rings . . . . . . . . . 109

Barry J. Gardner, Some aspects of T-nilpotence . . . . . . . . . . . . . . . 117

Juan A. Gatica and William A. Kirk, A fixed point theorem for $k$-set-contractions

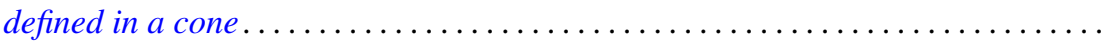

Kenneth R. Goodearl, Localization and splitting in hereditary noetherian prime

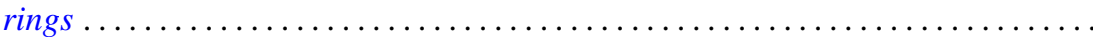

James Victor Herod, Generators for evolution systems with quasi continuous

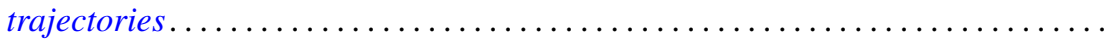

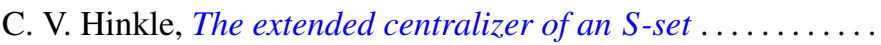

I. Martin (Irving) Isaacs, Lifting Brauer characters of p-solvable groups . . .

Bruce R. Johnson, Generalized Lerch zeta function ...........

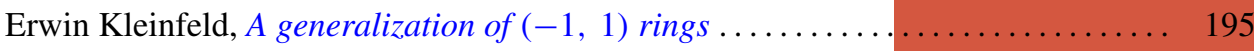

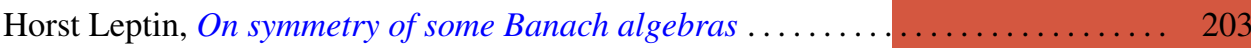

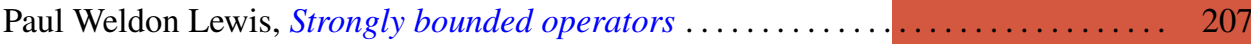

Arthur Larry Lieberman, Spectral distribution of the sum of self-adjoint

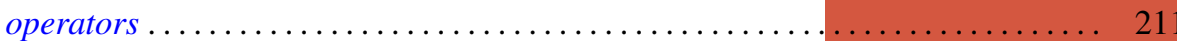

I. J. Maddox and Michael A. L. Willey, Continuous operators on paranormed spaces and matrix transformations

James Dolan Reid, On rings on groups ........................... 229

Richard Miles Schori and James Edward West, Hyperspaces of graphs are Hilbert cubes.

William H. Specht, A factorization theorem for p-constrained groups ...

Robert L Thele, Iterative techniques for approximation of fixed points of certain nonlinear mappings in Banach spaces ...............

Tim Eden Traynor, An elementary proof of the lifting theorem

Charles Irvin Vinsonhaler and William Jennings Wickless, Completely decomposable groups which admit only nilpotent multiplications .

Raymond O’Neil Wells, Jr, Comparison of de Rham and Dolbeault cohomology for

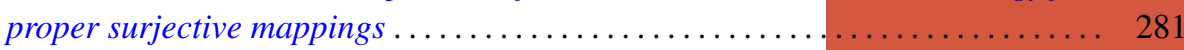

David Lee Wright, The non-minimality of induced central representations . . . . . 301 